\title{
THz Generation and Detection by Nonlinear Optical Methods
}

\author{
Sourabh Mukhopadhyay \\ Department of Physics, Jhargram Raj College (Govt. of West Bengal), Jhargram, INDIA \\ *Corresponding Author: smukhopadhyay.phy@gmail.com
}

Available online at: www.isroset.org

Received: 09/Mar/2018, Revised: 16/Mar/2018, Accepted: 29/Mar/2018, Online: 30/Apr/ 2018

\begin{abstract}
This article presents a comprehensive review on efficient $\mathrm{THz}$ generation by optical rectification and detection by electro-optic sampling. Starting with the basics of optical rectification and electro-optic field sensor, chronological development of the subject is presented. Performance of several zinc-blende crystals for THz generation as well as electrooptics sampling is discussed in detail and their comparative superiority relating to different crystals parameters are justified with experimental results. Organic crystals, due their higher nonlinearity, are important for $\mathrm{THz}$ generation by optical rectification and got special attention in this article. Phase-matching for $\mathrm{THz}$ generation by optical rectification is always a challenge and this issue is addressed with different schemes employed till date and optical rectification in tilted pulse front geometry is elaborated since it can dramatically increase the effective interaction length by achieving phase-matching. Material absorption in $\mathrm{THz}$ domain, which limits the $\mathrm{THz}$ generation efficiency, is however overcome by cryogenic cooling and performance of periodically poled lithium niobate under such low temperature for optical rectification in different schemes is presented in detail. The success in continuously increase of $\mathrm{THz}$ generation efficiency promises future high power $\mathrm{THz}$ devices for their application in basic science and technology. Recent propositions and theoretical models with predictions of orders of magnitude increase in the $\mathrm{THz}$ generation efficiency naturally pave the path with future challenges for the experimentalists. This article clearly figures out the directions of future research for $\mathrm{THz}$ generation by optical rectification and detection by electro-optic field sensor.
\end{abstract}

Keywords-Optical rectification, Difference frequency generation, Phase-matching, Electro-optic sampling,

\section{INTRODUCTION}

There has been a consistently growing research interest for last two decades, in the field of generation and manipulation of $\mathrm{THz}$ wave which occupies the frequency range from 0.1 to $10 \mathrm{THz}$ of the electromagnetic spectrum. This $\mathrm{THz}$ spectral range is of prime interest today due to it's wide spread application in science and technology, for example, in studying collective phonon dynamics, excitation of spin and electronic degree of freedom [1], free carrier absorption [2], time resolved spectroscopic and carrier dynamics studies [35], resonant control over material parameters [6] nonperturbative intraband response in antimonide [7], electron hole re-collision in semiconductor quantum structure [8], communication, security applications [9] and many more. Being a low energy non-ionizing radiation $\mathrm{THz}$ radiation is promising for future medical application, for example, in biomedical imaging [10,11], cancer detection [12], DNA repair and manipulation [13] etc. $\mathrm{THz}$ radiation with high field strength is also believed to be very promising to develop table top, compact charge particle accelerator [14].

There has been several approach for generating either few cycle broad band or multi-cycle narrow band $\mathrm{THz}$ radiation. The most conventional method was to excite biased photo- conductive switch by femtosecond (fs) laser pulse [15,16]. The photoconductive switch is essentially a semiconductor structure having two metal electrodes implanted on it. The choice of semiconductor material depends on the excitation laser wavelength. For Ti:Sapphire laser GaAs is a good choice since the band gap energy is less than the photon energy of the laser. The electrodes are made up of metals like gold or aluminum having separation $\sim 10 \mu \mathrm{m}$ and biased with voltage $10-50 \mathrm{~V}$ which produces an electric field $\sim$ few $\mathrm{kV} / \mathrm{cm}$. As the fs laser pulse is incident on the switch, free carriers are generated and are accelerated by the biased field. The rapid change in the polarization induced by the ultrafast laser generates coherent $\mathrm{THz}$ radiation which partially propagates along the electrodes and partially in the free space. Several geometry of photo conductive switches have been tried but the scope of this technique is limited for poor conversion efficiency and for not being able to be scaled to higher energies due to $\mathrm{THz}$ field saturation [17].

Another promising technique is to use gas plasma for generation of coherent $\mathrm{THz}$ radiation. In this technique an fs laser pulse of high pulse energy $(\sim \mathrm{mJ})$ and having central frequency $\omega$ is incident on a nonlinear crystal to produce second harmonic $(2 \omega)$ and then subsequently focussed on to a nobel gas to produce gas plasma. The fundamental and the 
second harmonic is mixed up in the gas plasma to produce directional electron current as well as coherent $\mathrm{TZ}$ radiation is by breaking the symmetry of the AC field [18-20]. Free electron laser could also be a good choice for generation of high power $\mathrm{THz}$ radiation [21], however, is expensive and thus access to such source is limited.

Most popular and promising technique for coherent THZ generation, till date, is to use nonlinear optical effect namely 'Optical rectification' (OR). This technique relies on series of difference frequency generation (DFG) between successive frequency components of an ultra-short broadband optical pulse. This technique is the most emerging one because of it's simplicity and capable of generating high power broad band single or multi-cycle coherent $\mathrm{THz}$ radiation. $\mathrm{THz}$ generation by $\mathrm{OR}$ was first demonstrated in zinc-blende crystal structure and later on many inorganic as well as organic crystals have been employed to achieve higher efficiency. Innovative approaches like tilting of incident pulse front have been tried and $\mathrm{THz}$ conversion efficiency was enhanced.

This paper aims to provide comprehensive review of coherent THZ generation by optical rectification [22,23]. Starting with the basics of the technique, a chronological development of the subject and the state of the art scenario is presented. Some experimental results have been reported here which gives a detail understanding on the THZ generation by OR. The major issues such as efficiency, choice of nonlinear crystal, excitation laser characteristics are addressed and different innovative modifications for producing high energy $\mathrm{THz}$ radiation have been discussed and compared in detail. Detection of $\mathrm{THz}$ radiation is another issue and therefore the technique of electro-optic sampling (EOS) in zinc-blende crystals has been discussed in detail $[24,25]$. The EOS has the potential to detect $\mathrm{THz}$ radiation irrespective of it's pulse energy, spectrum and cycle. Theory of EOS is presented along with experimental results obtained to identify the promise, challenges and also to address several issues with this technique.

\section{OPTICAL RECTIFICATION}

Optical rectification is essentially a second order nonlinear optical phenomenon first observed in 1962 [26]. If an intense laser pulse is incident on a second order nonlinear optical crystal it produces a dc polarization across the crystal by way of difference frequency generation [27] of the incident pulse spectrum. This phenomenon is exploited for generation of $\mathrm{THz}$ pulse from several crystals by use of a broad band optical pulse. An fs optical pulse has a broad bandwidth, however the frequency components are discrete by virtue of mode-locking. The separation between any two adjacent frequency components which lie in $\mathrm{THz}$ domain is constant and determined by the cavity round trip time of the laser oscillator. These closely spaced frequencies are rectified by way of DFG and results in producing an optical polarization for $\mathrm{THz}$ field. The $\mathrm{THz}$ polarization can be written as $[27,28]$

$$
P^{(2)}(\Omega)=\epsilon_{0} \int d_{i j k}^{(2)}(\Omega: \omega+\Omega ;-\omega) E_{j}(\omega+\Omega) E_{k}^{*}(\omega) d \omega
$$

Here $d^{(2)}$ represents the second order nonlinear coefficient tensor, , the electric filed and $\omega$ and $\Omega$ the optical and $\mathrm{THz}$ frequency respectively. However, for a polarized input fs pulse the electric field components will have the same polarization $(j=k)$. THz generation efficiency will depend choosing the geometry such that maximum value of $d$ tensor can be utilized. For zinc-blende crystals, under contracted notation of $d$ tensor, only three elements will be nonvanishing $\left(d_{14}=d_{25}=d_{36}\right)$ [29]. However, the THs efficiency will also depend crucially on phase matching of the optical and the $\mathrm{THz}$ wave. For $\mathrm{THz}$ generation as in (1), the phase matching condition is

$$
\Delta k=k(\omega+\Omega)-k(\omega)-k(\Omega)=0
$$

Here $k$ represents the wave vector. An equivalent quantity related to the phase matching is the coherence length, which determines the length of the crustal for which generation is possible and if we neglect the dispersion in the optical spectral range can be written as [22],

$$
l_{c}=\frac{\pi c}{\Omega\left|n_{o p t}-n_{T H z}\right|}
$$

Where $n_{\text {opt }}$ and $n_{T H z}$ are the refractive indices at the optical and the Thz frequency. Since the difference of the refractive indices at the $\mathrm{THz}$ and the optical frequency is very large the coherence length becomes very small. However, considering the dispersion in the optical range itself, many fold increase of the coherence length is possible. If we consider the dispersion in the optical spectrum the phase matching condition can be reduced to [22]

$$
\frac{k(\Omega)}{\Omega} \approx\left(\frac{\partial k}{\partial \omega}\right)_{o p t}
$$

This implies that in modified phase matching condition, the phase velocity $\left(v_{p}\right)$ of the $\mathrm{THz}$ wave should be equal to the group velocity $\left(v_{g}\right)$ of the optical pulse. The coherence length in such case can be brought in a form [22]

$$
l_{c}=\frac{\pi c}{\Omega\left|n_{o p t}-\lambda_{o p t}\left(\frac{d n_{o p t}}{d \lambda}\right)_{\lambda_{o p t}}-n_{T H z}\right|}
$$


In practice sufficient group velocity dispersion (GVD) exists in the optical domain. ZnTe, which is a very promising crystal for $\mathrm{THz}$ generation has sufficient GVD exists [29] around $800 \mathrm{~nm}$ such that phase matched $\mathrm{THz}$ around frequency of $2 \mathrm{THz}$ can be efficiently generated.

\section{ELECTRO-OPTIC SAMPLING}

Electro-optic sampling is an easy and effective way for detection of $\mathrm{THz}$ wave $[22,30,31]$. The process relies on the electric field induced birefringence of optical crystals. In this process the $\mathrm{THz}$ and a linearly polarized fs optical probe pulse is co-propagated in electro-optic (EO) crystal. The presence of $\mathrm{THz}$ field instantaneously modulates the index ellipsoid of the EO crystal and introduces a phase difference between the different components of the optical probe beam. The introduced phase difference can result in a polarization change of the probe beam. Therefore, if the output optical beam is analyzed with an analyzer, the electric field strength of the $\mathrm{THz}$ pulse can be figured out. Let us describe the process of EOS for the zinc-blende crystals, e.g. ZnTe. Zincblende crystals possess $\overline{4} 3 \mathrm{~m}$ point group symmetry and only three elements of the electro-optic tensor will be nonvanishing $\left(r_{14}=r_{25}=r_{36}\right)$. The crystal doesn't have birefringence; however, $\mathrm{THz}$ field can introduce birefringence in the crystal. Let us consider $\mathrm{THz}$ and the optical probe, both linearly polarized, are incident on the (110) plane of ZnTe crystal. The geometry is elaborated in Figure 1. $x[1,0,0], y[0,1,0$, and $z[0,0,1]$ represents the

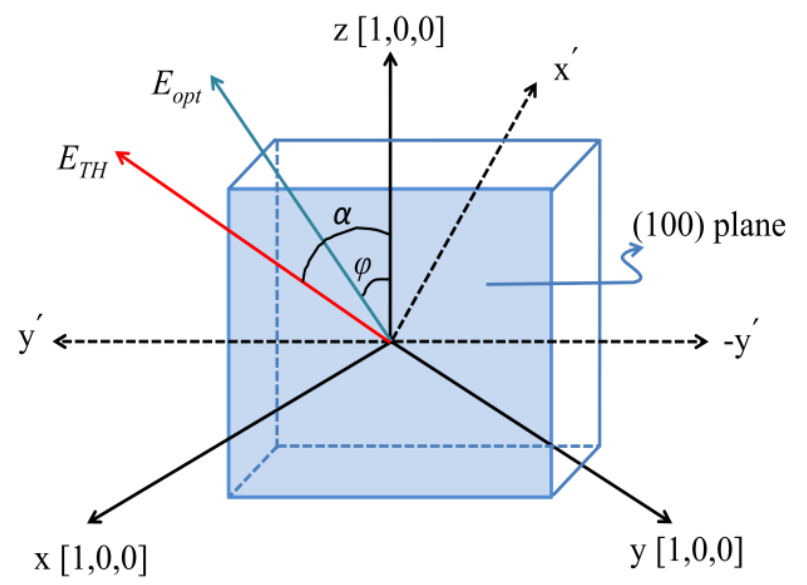

Figure 1. Geometry of (110) cut ZnTe crystal used as EOS of THz.

crystallographic axes of the ZnTe. The electric field of the $\mathrm{THz}$ wave $\left(E_{T H z}\right)$ and the electric field of the optical probe $\left(E_{\text {opt }}\right)$ make angles $\alpha$ and $\varphi$ respectively with the crystallographic $z$ axis. The non-birefringent ZnTe becomes birefringent under the influence of the $\mathrm{THz}$ field and the modified index ellipsoid becomes [30],

$$
\begin{aligned}
\frac{x^{2}+y^{2}+z^{2}}{n^{2}} & +2 E_{T H z, x} r_{41} y z+2 E_{T H z, y} r_{41} x z \\
& +2 E_{T H z, z} r_{41} x y=0
\end{aligned}
$$

Here $n$ is the unperturbed refractive index in absence of $\mathrm{THz}$ field. It should be noted that for the $\mathrm{THz}$ pulse propagating in (110) direction, $E_{T H z, x}=-E_{T H z, y}$. The presence of terms containing $y z, x z$ and $x y$ indicates that the principal axes of the modified index ellipsoid do not coincide with the crystallographic axes. However, new set of axes can be found so that the same would be the principal axes of the index ellipsoid. Let us make it with two successive coordinate transformations. Firstly, a rotation of axes by angle $45^{\circ}$ is made with suspect to the $z$ axis so that the new set of axes $\left(x^{\prime}, y^{\prime}, z^{\prime}\right)$ such that [31]

$$
\begin{gathered}
x=\frac{x^{\prime}}{\sqrt{2}}-\frac{y^{\prime}}{\sqrt{2}} \\
x=\frac{x^{\prime}}{\sqrt{2}}-\frac{y^{\prime}}{\sqrt{2}} \\
z=z^{\prime}
\end{gathered}
$$

With the help of the new transformed coordinate the index ellipsoid can be written as [31]

$$
\begin{gathered}
x^{\prime 2}\left(\frac{1}{n^{2}}+E_{T H z, Z}\right. \\
\left.r_{41}\right)+y^{\prime 2}\left(\frac{1}{n^{2}}-E_{T H z, z} r_{41}\right)+\frac{z^{\prime 2}}{n^{2}} \\
+2 \sqrt{2} E_{T H z, x} r_{41} y^{\prime} z^{\prime}=1
\end{gathered}
$$

Finally it is evident that a rotation around the $x^{\prime}$ axis is needed to align new coordinate $\operatorname{system}\left(x^{\prime \prime}, y^{\prime \prime}, z^{\prime \prime}\right)$ with the principal axes of the ellipsoid. Designating the required angle of rotation $\theta$, the transformation relation takes the form [31]

$$
\begin{gathered}
x^{\prime}=x^{\prime \prime} \\
y^{\prime}=y^{\prime \prime} \cos \theta-z^{\prime \prime} \sin \theta \\
z^{\prime}=y^{\prime \prime} \sin \theta+z^{\prime \prime} \cos \theta
\end{gathered}
$$

Noting that $E_{T Z, z}=E_{T H z} \cos \alpha$ and $E_{T H z, x}=-E_{T H z, y}=$ $\frac{E_{T H z}}{\sqrt{2}} \sin \alpha$; the finally transformed index of ellipsoid can be shown to have the form [32]

$x^{\prime \prime 2}\left(\frac{1}{n^{2}}+E_{T H} r_{41} \cos \alpha\right)$

$+y^{\prime \prime 2}\left\{\frac{1}{n^{2}}-E_{T H z} r_{41}\left[\cos \alpha \sin ^{2} \theta+\cos (\alpha+2 \theta)\right]\right\}$

$+z^{\prime \prime 2}\left\{\frac{1}{n^{2}}-E_{T H z} r_{41}\left[\cos \alpha \cos ^{2} \theta-\cos (\alpha+2 \theta)\right]\right\}$

$=1$

With the condition that

$2 \theta=-\arctan (2 \tan \alpha)-n \pi$ 
and $\left(n-\frac{1}{2}\right) \pi \leq \alpha \leq\left(n+\frac{1}{2}\right), n=0,1,2 \ldots \ldots$

If the electric field is not very strong, the refractive indices corresponding to propagation along $y^{\prime \prime}$ and $z$ " direction can be approximated as [31]

$$
\begin{aligned}
& n_{y^{\prime \prime}} \approx n+\frac{n^{3}}{2} E_{T H z} r_{41}\left[\cos \alpha \sin ^{2} \theta+\cos (\alpha+2 \theta)\right] \\
& n_{z^{\prime \prime}} \approx n+\frac{n^{3}}{2} E_{T H z} r_{41}\left[\cos \alpha \cos ^{2} \theta-\cos (\alpha+2 \theta)\right]
\end{aligned}
$$

Thus it is evident that the polarization component along $y^{\prime \prime}$ and $z$ " of the optical probe suffers different refractive indices and there will be an accumulated phase difference while propagating through the $\mathrm{ZnTe}$. The final polarization of the output optical polarization will be elliptic.

In experiment a balanced photo detector is placed after the EO crystal. The balanced photo detector comprises of a quarter-wave plate, Wollaston prism and two identical photodiodes. In absence of the $\mathrm{THz}$ field the linearly polarized optical wave becomes circularly polarized by the quarterwave plate and divided in two equal parts by the Wollaston prism. The two equal parts are fed into the two identical photo-diodes and the difference signal is monitored via Lock-in amplifier. In absence of the THz field the signal will be null. However in presence of the THz field, the incident beam to the quarter-wave plate becomes elliptical and finally the Wollaston prism splits the beam in two unequal parts, resulting in a difference signal in Lock-in amplifier. The difference signal can be shown to have the form [31]

$$
\begin{gathered}
\Delta S \propto I_{p} \frac{\omega^{3} n^{3} E_{T H z} r_{41} L}{2 c}(\cos \alpha \sin 2 \varphi \\
+2 \sin \alpha \cos 2 \varphi)
\end{gathered}
$$

Here $I_{p}$ is the probe intensity on the EO crystal and $L$ is the EO crystal length. The difference signal is traced as function of the delay between the probe and the $\mathrm{THz}$ pulse and it gives the actual replica of the THz field. It is evident that the signal can be maximized by rotating the EO crystal and the maximum signal can be obtained for $\varphi=\alpha+90$ or $\varphi=\alpha$.

\section{THZ GENERATION AND DETECTION}

The study of THz generation by OR started in 1990s. Rice et.al. studied $\mathrm{THz}$ generation in CdTe $\mathrm{InP}$ and GaAs crystals via OR [32]. They used Ti:Sapphire laser oscillator generating 200fs pulse train with $76 \mathrm{MHz}$ repletion rate and having fluence of $48 \mathrm{~nJ} / \mathrm{cm}^{2}$. The laser was incident normally on (110) crystallographic plane and the efficiency was maximized by varying the azimuthal angle of the crystals. Greater efficiency was observed for GaAs and reason was dispersion of second order susceptibility. They used a dipoler antenna as detector of the $\mathrm{THz}$ and the probe pulse from same laser was used to gate the emitted radiation $(\mathrm{THz})$ from zinc-blende crystals. The emission was detected by measuring the transient photo current but the wavelength not determined. In 1995, Bovalet et. al. studied $\mathrm{THz}$ generation exclusively in <110> GaAs crystal [33]. A Ti:sapphire oscillator delivering 10-15 fs pulses at a $100 \mathrm{MHz}$ repetition rate and wavelength spread from 0.72 to $0.88 \mathrm{~nm}$ was used to pump the GaAs crystal. Nitrogen cooled HgCdTe detector, which has a sharp cut off at $15 \mathrm{um}$ was used to detect the rectified signal and the emitted wavelength was not conclusively determined due to detector limitation. The proper detection of $\mathrm{THz}$ radiation by EOS was first demonstrated in 1996 by $\mathrm{Wu}$ et. al. [34]. The $\mathrm{THz}$ was generated by OR from GaAs crystal which was attached with metal coated glass. They used $\mathrm{LiTaO}_{3}$ crystal as electro-optic crystal for EO sampling and finally concluded that $\mathrm{ZnTe}$ demonstrated superior performance for free space EOS. Nahata et.al. described a wideband coherent $\mathrm{THz}$ spectroscopy system using optical rectification for the generation of $\mathrm{THz}$ radiation and electro-optic sampling for the coherent detection of this radiation. They used $\langle 110\rangle$ cut ZnTe crystal for both rectification and the EOS [22]. A mode-locked Ti:sapphire laser producing $130 \mathrm{fs}$ pulses at $800 \mathrm{~nm}$ with $76 \mathrm{MHz}$ repetition rate was used to pump $0.9 \mathrm{~mm}$ thick ZnTe crystal for $\mathrm{THz}$ generation by OR. Most important part of their experiment was that they succeeded to achieve phase-matching for the OR. Although they measured the frequency range $0-4 \mathrm{THz}$, the coherence length was found to exceed the crystal length of $0.9 \mathrm{~mm}$ for $\sim 2.5 \mathrm{THz}$ and therefore the higher frequency $\mathrm{THz}$ beyond $2.5 \mathrm{THz}$ was shown to have attenuated significantly. A broad bandwidth of \& $\mathrm{THz}$ and centered around $3.6 \mathrm{TH}$ was generated from GaAS crystal by OR and the same was detected by free space EOS using a GaP crystal [35]. A comparison was made and concluded that $r_{41}$ was found to be greater for $\mathrm{ZnTe}$ than $\mathrm{GaP}$. This observation certainly indicated the superiority of $\mathrm{ZnTe}$ among the zinc-blend crystals. A good comparison was made between $\mathrm{ZnTe}$, $c$-cut $\mathrm{LiNbO}_{3}$, and $b$-cut $\mathrm{LiTaO} 3$ in respect of their performance to be used as electro-optic sensor and it was found that signal to noise ratio was found to be orders of magnitude high for $\mathrm{ZnTe}$ [24]. This observation indicated the promise of $\mathrm{ZnTe}$ for a good electro-optic sensor. An innovative technique of using a quarter wave plate to produce extraordinary and ordinary polarization was demonstrated and the phase-matching for OR was achieved in GaSe crystal to generate bandwidth limited $\mathrm{THz}$ pulse [36]. It was shown that although two strong phase-matching peaks was observed at 8 and $25 \mathrm{THz}$, the presence of lattice resonance at $7.1 \mathrm{TH}$ the emission at $7.1 \mathrm{THz}$ was much narrower. GaSe become important for phase-matched $\mathrm{OR}$ and $\mathrm{THz}$ generation because of the inherent birefringence present in it [37] but it was difficult to process such crystal and the use was limited. ZeTe became more attractive in terms of availability, durability and performance for both OR and EOS. Ti:Sapphire laser pumped ZeTe, proved to be very promising for broad band, 
tunable source of $\mathrm{TH}$ radiation and with shaped pulse shape by liquid crystal modulator [38]. A typical set-up $\mathrm{THz}$ generation by OR and detection by EOS, based on ZnTe crystal, as we used in our experiment shown schematically in Figure 2. A Ti:Sapphire fs laser oscillator which provides length $75 \mathrm{~mm}$. The weak reflected part of the $808 \mathrm{~nm}$ radiation from the pellicle beam splitter is used as the probe beam. The probe beam is first passed through a computer

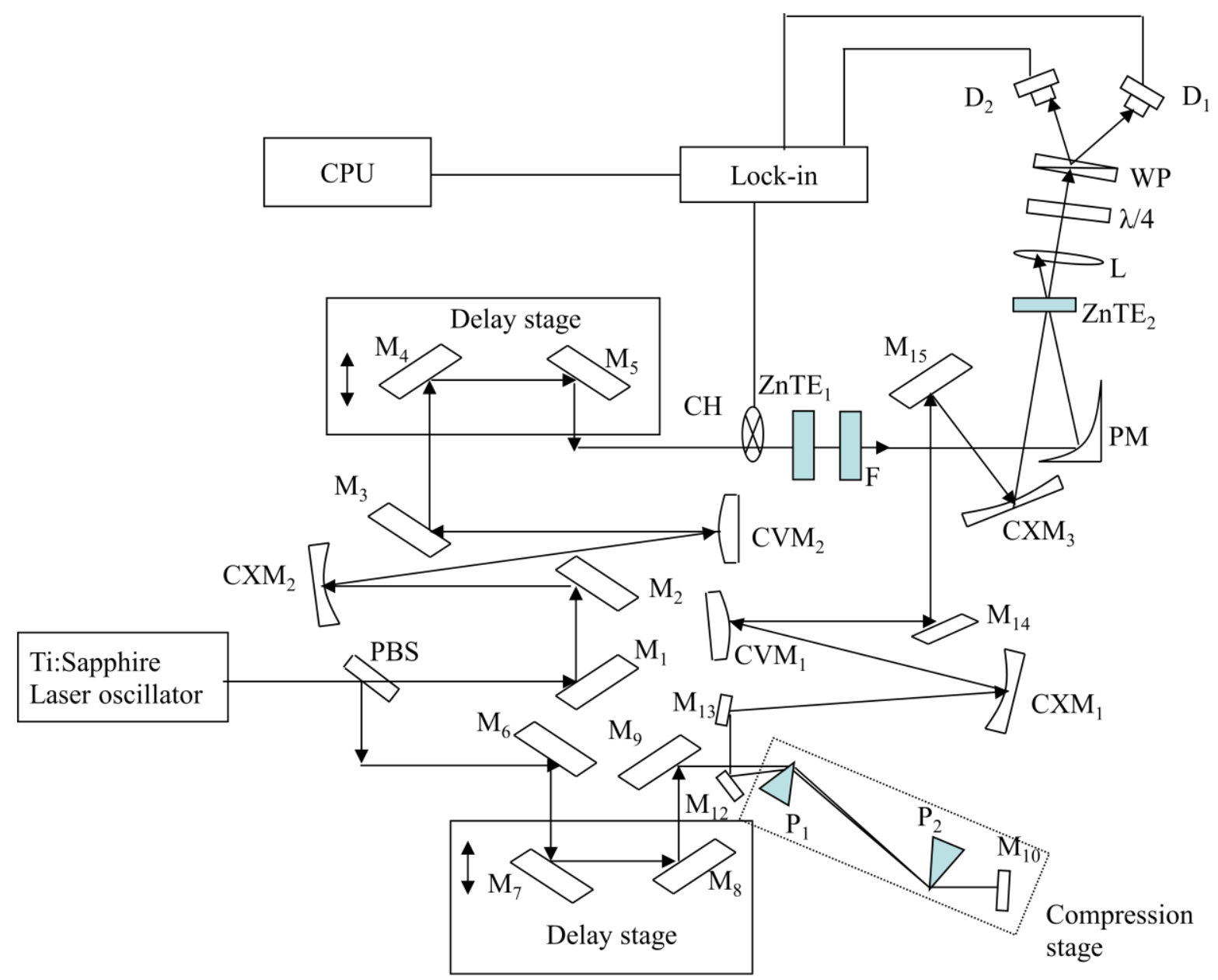

Figure 2. THz wave generation by nonlinear optical pulse rectification, and detection of the same by electro optic field sensor. PBS, Pellicle beam splitter; $\mathrm{M}_{1}$ .... $\mathrm{M}_{15}$, gold plated flat mirror. $\mathrm{CXM}_{1}$ and $\mathrm{CXM}_{2}$, plano-concave gold mirror of focal length $600 \mathrm{~mm} ; \mathrm{CVM}_{1}$ and $\mathrm{CVM}_{2}$, plano-convex gold mirror of focal length $250 \mathrm{~mm} ; \mathrm{P}_{1}$ and $\mathrm{P}_{2}$ right equilateral fused silica prism; ZnTe $1 \& \mathrm{ZnTe} 2$, <110> cut Zinc Telluride crystals ; F, Teflon filter; CH, chopper; $\lambda / 4$, quarter-wave plate; WP, Walston prism; PM, L, Convex lens of $50 \mathrm{~mm}$ focal length Gold coated off-axis parabola; D1 and D2 Si photo-detector.

$45 \mathrm{fs}$ pulses at $808 \mathrm{~nm}$ with $100 \mathrm{MHz}$ repetition rate was used in this experiment. The average laser power of $40 \mathrm{~mW}$ was available for the experiment. A Pellicle beam splitter of $\mathrm{R}: \mathrm{T}$ of $8: 92$ is used to split the beam in two parts. The transmitted stronger part is collimated by using a concave and a convex mirror of focal length $600 \mathrm{~mm}$ and $250 \mathrm{~mm}$ respectively and it is used as the pump for THz generation. A $<110>$ cut ZnTe crystal of thickness $500 \mu \mathrm{m}$ is pumped by the pump beam to generate $\mathrm{THz}$ wave after. The residual 808 $\mathrm{nm}$ radiation is filtered out for the $\mathrm{THz}$ wave using a teflon filter and the $\mathrm{THz}$ wave is then focused on a ZnTe crystal (ZnTe2) by using a gold plated off-axis parabola of focal controlled delay stage, and then a compression stage formed by two equilateral prisms made of fused silica. The compressed pulse width as measured by second order autocorrelation using two-photon absorption diode is found to be $24 \mathrm{fs}$. The compressed probe pulse is then collimated by using a concave and a convex mirror of focal length $600 \mathrm{~mm}$ and $250 \mathrm{~mm}$ respectively d finally focused on the $\mathrm{ZnTe}$ crystal. The angle between the pump and the probe beam is kept under 5 degree. The spatial overlapping between pump and the probe beam on the $\mathrm{ZnTe}$ crystal is assured by using a pinhole. The $\mathrm{THz}$ wave modifies the refractive indicatrix of the $\mathrm{ZnTe}$ crystal. The refractive index 
value along the axis parallel to the $\mathrm{THz}$ electric field is modified due to the Pockel's effect, while the refractive index along the axis perpendicular to the $\mathrm{THz}$ electric filed remains unaltered. As such, under the influence of the $\mathrm{THz}$ field the ZnTe crystal becomes birefringent, which changes the polarization state of the probe beam. First the $\mathrm{THz}$ wave is blocked, and the linearly polarized probe beam, after passing through the ZnTe crystal is incident on a quarter wave plate, followed by a Wollaston prism. The quarter wave plate makes the probe beam to be circularly polarized. The circularly polarized beam is then spited in two equal parts of linearly polarized beam by a Wollaston prism and is detected by two Si photo-diodes connected to lock-in amplifier in differential mode. When $\mathrm{THz}$ wave is not
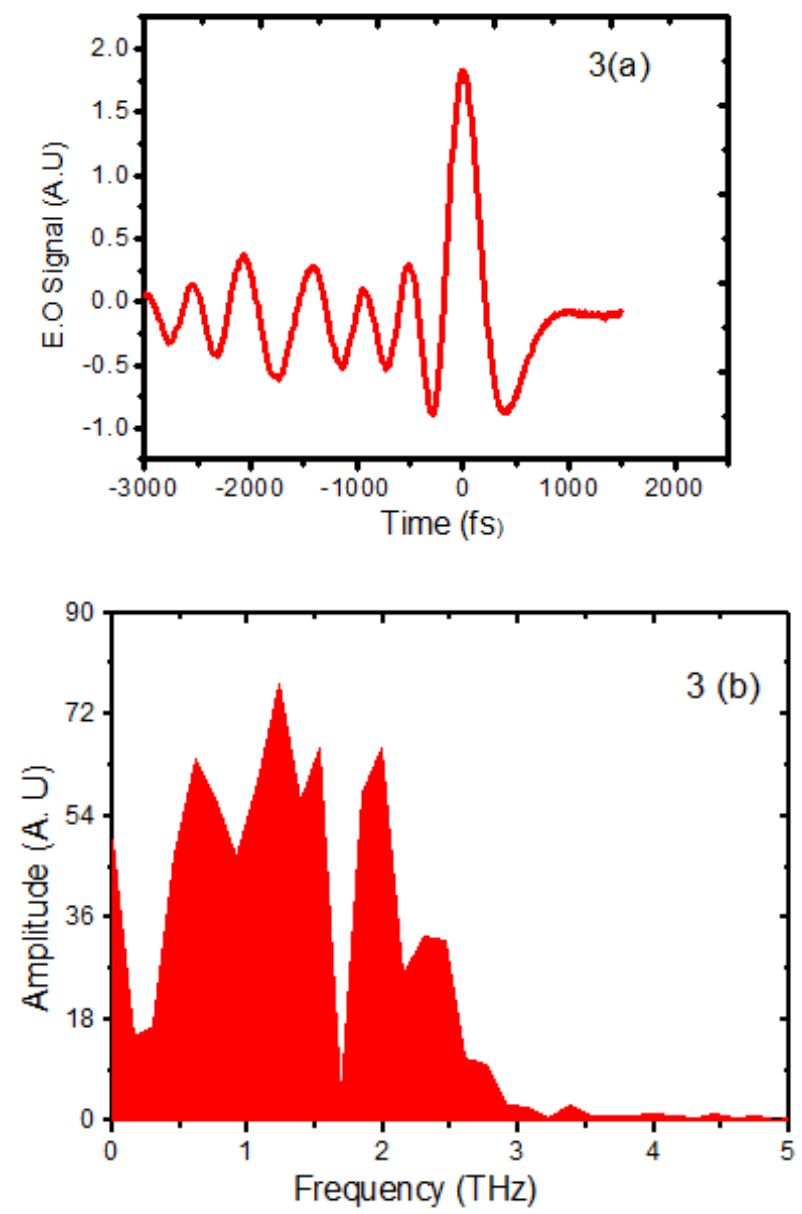

Figure 3. (a) electro-optic signal detected by $200 \mu \mathrm{m}$ thick (110) $\mathrm{ZnTe}$ crystal; (b) Corresponding THz spectrum obtained by FFT of (a)

present, the two photo-diodes are balanced. Now the $\mathrm{THz}$ wave is made to incident on the $\mathrm{ZnTe}$ sensor and is temporally overlapped with the probe beam by using the
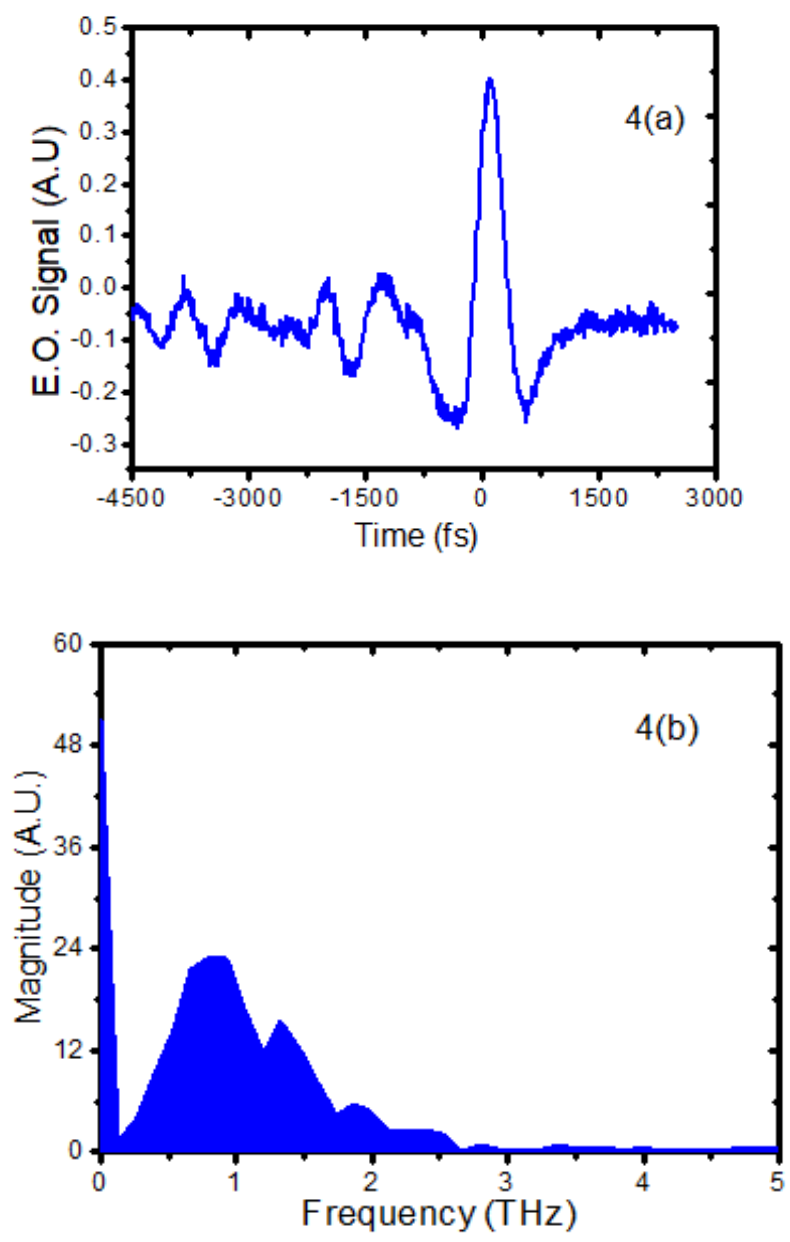

Figure 4. (a) electro-optic signal detected by $20 \mu \mathrm{m}$ thick (110) ZnTe crystal; (b) Corresponding THz spectrum obtained by FFT of (a)

delay stage. Under the action of the $\mathrm{THz}$ field the polarization of the probe beam becomes slightly elliptical and the two photo-diodes become unbalanced. The unbalanced signal is detected through a lock-in amplifier, which is triggered by the signal from chopper used on the pump beam. The cross-correlation signal is thus obtained as a function of time as the probe is scanned over the $\mathrm{THz}$ beam in temporal domain. Two detection crystals have been used to detect the electro-optic signal. Figure 3(a) shows the electro-optic signal detected for a ZnTe (ZnTe2) crystal of thickness $200 \mu \mathrm{m}$. The Fourier spectrums of the THz field envelop which shows the frequency spectrum of the $\mathrm{THz}$ radiation is shown in Figure 3(b). Electro-optic signal for 10 $\mu \mathrm{m} \mathrm{ZnTe}$ sampling crystal is shown in Figure. 4 (a) and corresponding frequency spectrum is shown in Figure 4(b). The reduction in sampling crystal thickness increases the signal to noise ratio. It also helps reducing the group velocity mismatch between the $\mathrm{THz}$ and the probe pulse and thus the 
larger bandwidth of the THz pulse can be detected. Since the cross-correlation signal is taken as the ensemble average over a large number of pulses the reconstruction of the replica of the $\mathrm{THz}$ electric field is possible only when the carrier envelope phase of the pulse stable from pulse to pulse. It thus proves the stability of the carrier envelope phase in a rectification process and is very useful to study ultrafast charge carrier dynamics and generation. The carrier envelope phase can also be measured precisely by the charge symmetry produced air plasma [39]. The choice of thickness of the crystal is very important for generating efficient $\mathrm{THz}$ by OR. Very thin crystal can reduce the efficiency and on the other hand a thick crystal can reduce the phase-matching band-width, truncate the spectrum and even can split the $\mathrm{THz}$ pulse in time domain [40]. However, more recently, in another report it was shown that thick crystals exhibited a flatter frequency response and since they are mechanically more stable, cheaper, and easier to provide and to handle, Thick crystal could be suitable for EO sensor [41]. Kapfarth el.al. showed that $250 \mu \mathrm{m}$ thick (110) cut ZnTe is as efficient as $10 \mu \mathrm{m}$ for electro-optic detection of 0.3 to50 $\mathrm{THz}$ domain [41].

Increase in the efficiency of $\mathrm{THz}$ generation became a great concern and several methods, crystals and engineered crystals also came in use. Imeshev et. al. used quasi-phasematching (QPM) to increase the interaction length and thereby to increase the efficiency of the $\mathrm{THz}$ generation [42]. They generated $3.3 \mu \mathrm{W}$ average power, $100-\mathrm{MHz}$ repetition rate source in the range of $1.78-2.49 \mathrm{THz}$ from orientation patterned GaAs pumped by a compact all-fiber femtosecond laser at the wavelength of $2 \mathrm{um}$. Lee et. al. demonstrated the generation of multi-cycle narrow-bandwidth terahertz radiation based optical rectification in periodically inverted GaAs structures. Three different structures: optically contacted multilayer, orientation-patterned, and diffusionbonded GaAs were used and it was possible to generate tunable 0.8 to $3 \mathrm{THz}$ radiation by use of different structure periods [43]. Large aperture ZnTe was also used to increase the $\mathrm{THz}$ generation efficiency energies up to $1.5 \mu \mathrm{J}$ per pulse and a spectral range extending to $3 \mathrm{THz}$ were obtained using a $100 \mathrm{~Hz} \mathrm{Ti:sapphire} \mathrm{laser} \mathrm{source} \mathrm{and} \mathrm{a} \mathrm{75-mm} \mathrm{diameter,}$ 0.5-mm-thick, (110) cut ZnTe crystal, corresponding to an average power of $150 \mu \mathrm{W}$ and an energy conversion efficiency of $3.1 \times 10^{-5}$ [44]. Another very popular crystal Lithium niobate came up to produce pulses of $125 \mu \mathrm{J}$ energy and with the $0.25 \%$ efficiency were generated by OR of 1.3 ps pulses [45]. Experimental findings together with theoretical predictions indicated the feasibility of efficient $\mathrm{THz}$ pulse generation with $\mathrm{mJ}$-level output energy by using optimal pump pulse duration, cooling the Lithium niobate crystal, and using a contact grating.

Becuase of higher nonlinear coefficient, organic crystal attracted attention in the field of $\mathrm{THz}$ generation by $\mathrm{OR}$. Broadband terahertz pulses in the frequency range 0.5 and 2.5 THz, have been generated in 2-cyclooctylamino-5- nitropyridine (COANP) single crystals by optical rectification of 150 fs laser pulses from an amplified Ti:Sapphire laser [23]. Fabian et. al. claimed COANP to be more efficient than ZnTe for THZ generation by OR. THz radiation is generated by $\mathrm{OR}$ in an organic salt crystal 4N,Ndimethylamino-4'-N'-methyl stilbazolium tosylate, called DAST, pumped with the signal wavelength of a powerful optical parametric amplifier [46]. Conversion efficiencies up to $2 \%$ are reported and carrier envelope phase (CEP) stabilized $\mathrm{THz}$ pulses at a center frequency of $2.1 \mathrm{THz}$ with a bandwidth of $2 \mathrm{THz}$ was generated. $1.5 \%$ pump to $\mathrm{THz}$ energy conversion efficiency was reported and single-cycle broadband terahertz pulses with a central frequency of 1.5 $\mathrm{THz}$ was generated by OR from organic salt crystal 2-[3-(4hydroxystyryl) -5.5 - dimethyl cyclohex -2 - enylidene] malononitrile or $\mathrm{OH} 1$ [47]. High-field THZ single-cycle pulses with 1:5 MV/cm are generated by optical rectification in the stilbazolium salt crystal 4-N,N-dimethylamino-4'-N'methyl-stilbazolium 2,4,6-trimethyl benzenesulfonate (DSTMS) with conversion efficiency of $0.8 \%$ [48]. In another report $\mathrm{THz}$ conversion efficiency of $3.2 \%$ and $\mathrm{THZ}$ pulse energy of $270 \mu \mathrm{J}$ claimed to be obtained from $\mathrm{OH} 1$ crystal [49].

In order to select proper crystal for $\mathrm{THz}$ generation by $\mathrm{OR}$ a figure of merit (FOM) was introduced. The FOM of a crystal for THZ generation without considering the absorption loss is defined as [50]

$$
F O M \equiv \frac{d_{e f f}^{2}}{n_{o p t}^{2} n_{T H z}}
$$

And the same but considering the absorption loss is defined as

$$
F O M_{A} \equiv \frac{d_{e f f}^{2}}{n_{o p t}^{2} n_{T H z} \alpha_{T H z}^{2}}
$$

Here $\alpha_{T H z}$ represents the absorption coefficient in THZ domain. Table 1 summarizes crystal parameters and FOM of some selected crystals corresponding to pump wavelength of $800 \mathrm{~nm}[50]$.

Here $r$ represents the electro-optic coefficient, $n_{\text {opt }}$; the refractive index at optical wavelength and $n_{o p t}^{g r}$; the group refractive index of optical pulse.

The organic crystals, due to their higher nonlinearity seemed to be attractive however is not always suitable for nonlinear optics in general. The crystals are fragile, susceptible to atmosphere and therefore difficult to handle in experimental conditions. $\mathrm{LiNbO}_{3}$, on the other hand is very popular due it's robustness, higher nonlinearity, availability of god quality large crystals and high damage threshold. The challenges of $\mathrm{THz}$ generation in $\mathrm{LiNbO}_{3}$ are two-fold; $\mathrm{THz}$ absorption and difficulty to achieve phase-matching. The refractive indices of $\mathrm{LiNbO}_{3}$ at optical frequencies is $\sim 5$ 
Table1. Properties of some crystals and their merit for THz generation by OR

\begin{tabular}{|l|c|c|c|c|c|c|c|c|}
\hline Crystal & $\begin{array}{c}r \\
(p m / V)\end{array}$ & $\begin{array}{c}d \\
(\mathrm{pm} / \mathrm{V})\end{array}$ & $n_{\text {opt }}$ & $n_{\text {opt }}^{\mathrm{gr}}$ & $n_{\mathrm{THz}}$ & $\begin{array}{c}\alpha_{T H z} \\
\left(\mathrm{~cm}^{-1}\right)\end{array}$ & FOM $\left(\frac{p^{2}}{V^{2}}\right)$ & $F_{\text {OM }}\left(\frac{\mathrm{pm}^{2} \mathrm{~cm}^{2}}{V^{2}}\right)$ \\
\hline $\mathrm{GaAS}$ & 1.43 & 65.6 & 3.68 & 4.18 & 3.61 & 0.5 & 87.9 & 352 \\
\hline $\mathrm{GaP}$ & 0.97 & 24.8 & 3.18 & 3.57 & 3.34 & 1.9 & 18.2 & 5.0 \\
\hline $\mathrm{ZnTe}$ & 4.04 & 68.5 & 2.87 & 3.31 & 3.17 & 1.3 & 180 & 106 \\
\hline $\mathrm{GaSe}$ & 1.7 & 28.0 & 2.85 & 3.13 & 3.72 & 0.07 & 25.9 & 5300 \\
\hline $\mathrm{LiTaO}_{3}$ & 30.5 & 161 & 2.145 & 2.22 & 6.42 & 46 & 882 & 0.4 \\
\hline $\mathrm{LiNbO}_{3}$ & 30.9 & 168 & 2.159 & 2.23 & 5.16 & 16 & 1170 & 4.6 \\
\hline $\mathrm{DAST}$ & 77 & 618 & 2.38 & 3.31 & 2.4 & 150 & 28000 & 1.2 \\
\hline
\end{tabular}

while it is $\sim 2$ for $\mathrm{THz}$ frequency range. The large difference in the refractive indices makes it difficult to achieve birefringence phase-matching for $\mathrm{THz}$ generation in $\mathrm{LiNbO}_{3}$. To counter this problem, an innovative technique of $\mathrm{OR}$ using tilted pulse-front (TPF) of the optical pump pulse was introduced and significant enhancement in the $\mathrm{THz}$

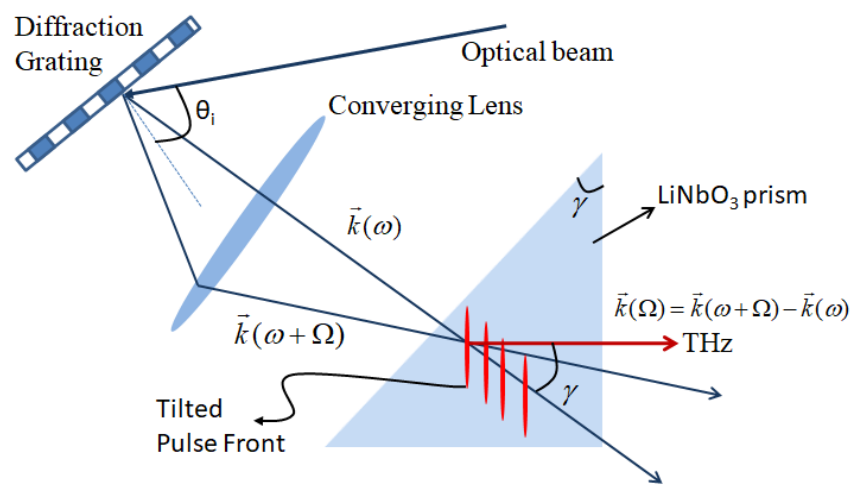

Figure 5. Geometry and phase-matching scheme in Tilted pulse front geometry in $\mathrm{LiNbO}_{3}$

generation efficiency was achieved [50-57]. The principle of OR using TPF is depicted in Figure 5. In this approach the broad band optical pump pulse is incident on a reflection grating and gets angularly dispersed. Different frequencies of the optical pulse would be dispersed in different angles and in Figure 5 two such wave vectors $\vec{k}(\omega)$ and $\vec{k}(\omega+\Omega)$ are shown. The angularly dispersed optical beam is imaged on to a prism shaped $\mathrm{LiNbO}_{3}$ crystal by use of a converging lens. The dispersed beam has the phase front normal to the propagation direction, however, the intensity front gets tilted with respect to the phase front by an angle say $\gamma$. The generated $\mathrm{THz}$ beam emerges at an angle $\gamma$ with respect to the direction of propagation of the optical beam. The apex angle of the prism is chosen close $\gamma$ so that $\mathrm{THz}$ exits normally from the face of the prism. The phase-matching condition in $\mathrm{LiNbO}_{3}$ prism for OR remains same as (2), however, since the optical beam and the $\mathrm{THz}$ travels different distance in $\mathrm{LiNbO}_{3}$ crystal within same time, phase-matching is achieved. The velocity matching condition under TPF geometry can be written as

$$
v_{o p t}^{g} \cos \gamma=v_{T H z}^{p}
$$

It should be remembered that in $\mathrm{LiNbO}_{3} \quad v_{o p t}^{g}>v_{T H z}^{p}$, but presence of the tilt in the intensity front of the optical pulse makes it possible to achieve phase-matching. The experimentally observed $\mathrm{THz}$ generation efficiency was far below that predicted theoretically in case of TPF geometry. The reason of such difference in theoretical and experimentally observed efficiency is the following. There is an spectral broadening of the optical pulse associated with the $\mathrm{THz}$ generation and this happens due to cascaded down frequency shift of the optical pulse. Due to this large spectral broadening dispersive effect gets emphasized and the conversion efficiency drops with the propagation $[58,59]$. However, in comparison to the traditional OR, the $\mathrm{THz}$ efficiency was increased notably in TPF geometry. $\mathrm{THz}$ generation efficiency varying from $0.25 \%$ to $1.15 \%$ was observed at room temperature by OR in TPF geometry [54-57,60]. The $\mathrm{THz}$ absorption was almost eliminated by cryogenically cooling and a conversion efficiency of $3.7 \%$ was recorded in congruent $\mathrm{LiNbO}_{3}$ [61].

Different schemes are coming up today to increase the $\mathrm{THz}$ generation efficiency. Recent theoretical study based on cascaded parametric amplification predicts conversion efficiency more than $8.0 \%$ is possible in cryogenically cooled periodically poled $\mathrm{LiNbO}_{3}$ [62]. This scheme relies on multi cycle low bandwidth pump and seed optical pulse, shifted in frequency by $0.5 \mathrm{THz}$. The pump and the seed initiate the narrow band $\mathrm{THz}$ generation in periodically poled $\mathrm{LiNbO}_{3}$ by $\mathrm{DFG}$ and the generated $\mathrm{THz}$ then simultaneously drives the repeated generation of optical lines. The cascaded generation of the optical lines are red shifted by the $\mathrm{THz}$ frequency and self start consecutive dramatic cascading process. This process shows exponential growth of $\mathrm{THz}$ energy growth over a single pass through a single crystal. In another more practically viable approach uses laser pulse sequences to drive the cascaded difference frequency generation of high energy, high peak-power and multi-cycle terahertz pulses in cryogenically cooled 
periodically poled lithium niobate [63]. Conversion efficiency more than $10 \%$ has been projected to achieve through this approach. However, both the approaches need experimental verification in future days.

\section{CONCLUSION AND FUTURE SCOPE}

Optical rectification has been shown to be an efficient way for generating either single cycle broad band or muti-cycle narrow band $\mathrm{THz}$ radiation. This process generates carrier envelope phase stable $\mathrm{THz}$ and therefore electro-optic sampling is the best technique for detection of such $\mathrm{THz}$ pulse. In optical rectification, optical frequency components get rectified by way of DFG in a nonlinear optical crystal and a low frequency $\mathrm{THz}$ field is generated. In the initial stages zinc-blende crystals, like $\mathrm{ZnTe}, \mathrm{GaAs}$ have been used for $\mathrm{THz}$ generation by OR, however getting notable $\mathrm{THz}$ generation efficiency and achieving phase-matching were the challenges to overcome. The phase-matching, which in this case implies the matching of the phase velocity of the $\mathrm{THz}$ pulse and the group velocity of the optical pump pulse, was difficult to achieve due to large difference of the refractive indices in optical and $\mathrm{THz}$ domain. This limits the effective crystal length over which the nonlinear interaction can take place. Consideration of the dispersion of the optical spectrum itself could increase the interaction length and the method OR became promising for efficient $\mathrm{THz}$ generation. Several zinc-blende crystals and different pump pulse width have been tried in many innovative experimental configurations, but the efficiency of $\mathrm{THz}$ generation was limited to a maximum value $\sim 0.1 \%$. However, large bandwidth of the $\mathrm{THz}$ pulse was possible to generate. The employment of organic crystals, owing to their large nonlinearity, could push up the conversion efficiency up to $\sim 0.5 \%$. An innovative idea of tilting the intensity front of the optical pump pulse by way of angular dispersion through the use of a grating and then imaging the same on a $\mathrm{LiNbO}_{3}$ prism became a very effective and efficient way to achieve phase-matching. This tilted pulse front geometry was able to generate high energy $\mathrm{THz}$ pulse with efficiency $\sim 1 \% \cdot \mathrm{LiNbO}_{3}$ became a promising candidate for $\mathrm{THz}$ generation because of its high nonlinearity, durability, availability of large crystals with good optical quality and high damage threshold, however, the absorption in the $\mathrm{THz}$ spectral domain restricted the effective crystal length and the efficiency of $\mathrm{THz}$ conversion. Cryogenic cooling of $\mathrm{LiNbO}_{3}$ gave a breakthrough to suppress the absorption and an efficiency more 3\% has been recorded. New propositions like cascaded parametric amplification and use of laser pulse sequences for cascaded DFG in periodically poled Lithium niobate, are coming up which claims the more than $10 \%$ $\mathrm{THz}$ generation efficiency is possible in near future. The field of high energy $\mathrm{THz}$ generation is becoming more vibrant owing to its tremendous versatile use in basic science and in technology.

\section{REFERENCES}

[1] S. J. B. Baxter and G. W. Guglietta, "THz Spectroscopy", Analytical Chemistry, Vol. 83, Issue 10, pp. 4342-4386, 2011.

[2] C. Ndebeka-Bandou, M. Rosch, K. Ohtani, M. Beck, and J. Faist, "Negative free carrier absorption in terahertz quantum cascade lasers", Applied Physics Letters, Vol. 108, Issue 9, pp. 0911029(13), 2016.

[3] R. Ulbricht, E. Hendry, J. Shan, T. F. Heinz, M. Bonn, “Carrier dynamics in semiconductors studied with time-resolved terahertz spectroscopy", Review of Modern Physics, Vol.83, Issue 2, pp. 543-586, 2011

[4] H. Hirori, K Tanaka, "Nonlinear Optical Phenomena Induced by Intense Single-Cycle Terahertz Pulses", IEEE J. Sel. Top. Quantum Electron., Vol. 19, Issue 1, pp. 8401110 (10), 2013

[5] D. Tsokkou, A. Othonos, and M. Zervos, "Carrier dynamics and conductivity of $\mathrm{SnO} 2$ nanowires investigated by time-resolved terahertz spectroscopy", Applied Physics Letters, Vol. 100, Issue 13, pp. 133101(1-3), 2012

[6] T. Kampfrath, K.Tanaka and K. A. Nelson, "Resonant and nonresonant control over matter and light by intense terahertz transients", Nature Photonics, Vol. 7 , Issue 9, pp. 680-690, 2013.

[7] F. Junginger, B. Mayer, C. Schmidt, O. Schubert, S. Mährlein, A. Leitenstorfer, R. Huber, and A. Pashkin, "Nonperturbative Interband Response of a Bulk InSb Semiconductor Driven Off Resonantly by Terahertz Electromagnetic Few-Cycle Pulses", Physical Review Letters, Vol. 109, Issue 14, pp. 147403(1-5), 2012.

[8] B. Zaks, R. B. Liu, M.S. Sherwin, "Experimental observation of electron-hole re-collisions", Nature, Vol. 483, Isse 7391, pp. 580-583, 2012.

[9] M. Tonouch, "Cutting-edge terahertz technology" , Nature Ptotonics, Vol. 1, Issue 2, pp. 97-105, 2007

[10] A. Redo-Sanchez, B. Heshmat, A. Aghasi, S. Naqvi, M. Zhang, J. Romberg and R. Raskar, "Terahertz time-gated spectral imaging for content extraction through layered structures", Nature Communications., Vol. 7, Issue 12665, pp. 1-7, 2106.

[11] M. Jewariya, E. Abraham, T. Kitaguchi, Y. Ohgi, M. Minami, T. Araki and T. Yasui, "Fast three-dimensional terahertz computed tomography using real-time line projection of intense terahertz pulse", Optics Express, Vol. 21, Issue 2, pp 2423-2433, 2013.

[12] P. C. Ashworth, E. Pickwell-MacPherson, E. Provenzano, S. E. Pinder, A. D. Purushotham, M. Pepper, and V. P. Wallace, "Terahertz, pulsed spectroscopy of freshly excised human breast cancer," Opt. Express, Vol. 17, Issue 15, pp. 12444-12454, 2009.

[13] L. V. Titova, A. K Ayesheshim, A. Golubov, D. Fogen, R. Rodriguez-Juarez, F.A. Hegmann, O. Kovalchuk, "Intense $\mathrm{THz}$ pulses cause H2AX phosphorylation and activate DNA damage response in human skin tissue", Biomedical Optics Express, Vol. 4, Issue 4, pp. 559-568, 2013.

[14] E. A. Nanni, W. R. Huang, K. Hong, K. Ravi, A. Fallahi, G. Moriena, R. J. D. Miller and F. X. Ka"rtner, THz accelerator, "Terahertz-driven linear electron acceleration", Nature Commun., Vo. 6, Issue 8486, pp. 1-8, 2015.

[15] Z. D. Taylor, E. R. Brown, J. E. Bjarnason, M. P. Hanson, and A. C. Gossard, "Resonant-optical-cavity photoconductive switch with $0.5 \%$ conversion efficiency and $1.0 \mathrm{~W}$ peak power", Optics Letters, Vol. 31, Issue 11, pp. 1729-1731, 2006.

[16] Y. C. Shen, P. C. Upadhya, E. H. Linfield H. E. Beere, "Ultrabroadband terahertz radiation from low-temperature-grown 
GaAs photoconductive emitters", Applied Physics Letters, Vol. 15, Issue 83, pp. 3117-3119, 2003.

[17] D. H. Auston, P. R. Smith, "Generation and Detection of Milimeter Waves by Picosecond Photoconductivity", Applied Physics Letters, Vol. 43, Issue 7, pp. 631-633, 1983.

[18] H. Hamster, A. Sullivan, S. Gordon, W. White and R. W. Falcone, "Subpicosecond, electromagnetic pulses from intense laser-plasma interaction" Physical Review Letters, Vol. 71, Issue 17, pp. 2725-2728, 1993.

[19] K. Y. Kim, A. J. Taylor, J. H.Glownia and G. Rodriguez, "Coherent control of terahertz supercontinuum generation in ultrafast laser-gas interactions" Nature Photonics, Vol. 2, Issue 7, pp. 605-609, 2008.

[20] J. M. Dai, N. Karpowicz and X. Zhang, "Coherent Polarization Control of Terahertz Waves Generated from Two-Color LaserInduced Gas Plasma", Physical Review Letters, Vol. 103, Issue 2,pp. 023001(1-4), 2009

[21] S. V. Benson, D. R. Douglas, P. Evtushenko, F. E. Hannon, C. Hernandez-Garcia, J. M. Klopf, R. A. Legg, G. R. Neil, M. D. Shinn, C. D. Tennant, S. Zhang and G. P. Williams, "Photon Source Capabilities of the Jefferson Lab FEL", Journal of Physics: Conf. Series, Vol. 425, Issue 7, pp 072002 (1-5), 2013.

[22] A. Nahata, A. S. Weling and T. F. Heinz, "A wideband coherent terahertz spectroscopy system using optical rectification and electro-optic sampling", Applied Physics Letters, Vol. 69, Issue 16, pp. 2321-2323, 1996

[23] F. D. J. Brunner, A. Schneider, and P. Günter, "Velocity-matched terahertz generation by optical rectification in an organic nonlinear optical crystal using a Ti:sapphire laser, Applied Physics Letters, Vol. 94, Issue 6, pp. 061119(1-3), 2009

[24] C. Winnewisser, P. U. Jepsen, M. Schall, V. Schyja, and H. Helm, "Electro-optic detection of $\mathrm{THz}$ radiation in $\mathrm{LiTaO}, \mathrm{LiNbO}_{3}$ and ZnTe", Applied Physics Letters, Vol. 70, Issue 23, pp. 30693071, 1997.

[25] P. C. M. Planken, H. K. Nienhuys H. J. Bakker and T. Wenckebach, "Measurement and calculation of the orientation dependence of terahertz pulse detection in ZnTe", Journal Optical. Society of America B, Vol. 18, No. 3, pp. 313-317. 2001.

[26] M. Bass, P. A. Franken, J. F Ward and G. Weinreich, "Optical rectification", Physical Review Letters, Vol. 9, Issue 11, pp. 446$448,1962$.

[27] R. W. Boyd, Nonlinear Optics $2^{\text {nd }}$ Ed., Academic Press, 2003.

[28] N. Bloembergen, Nonlinear Optics, (Bejamin, New York, 1965.

[29] V. G. Dmitriev, G. G. Gurzadyan and D. N. Kikogosyan, "Hand book of nonlinear optical crystals $3^{\text {rd }}$ Ed", Springer-Verlag, Germany, 1999.

[30] Q. Chen, M. Tani, Zhiping Jiang, and X.-C. Zhang, "Electro-optic transceivers for terahertz-wave applications", Journal of Optical Society of America B, Vol. 18, No. 6, pp. 823-831, 2001.

[31] P. C. M. Planken, H. K. Nienhuys and H. J. Bakker, T. Wenckebach, "Measurement and calculation of the orientation dependence of terahertz pulse detection in ZnTe", Journal of Optical Society America B Vol. 18, Issue 3, ,pp. 313-317, 2001.

[32] A. Rice,Y. Jin, X. F. Ma, X.-C. Zhang, D. Bliss, J. Larkin, and M. Alexander, "Terahertz optical rectification from $\langle 110\rangle$ zincblende crystals", Applied Physics Letters, Vol. 64, Issue 11, pp. 1324-1326, 1994.

[33] A. Bonvalet, M. Joffre, J. L. Martin and A. Migus, "Generation of ultrabroadband femtosecond pulses in the mid-infrared by optical rectification of $15 \mathrm{fs}$ light pulses at $100 \mathrm{MHz}$ repetition rate", Applied Physics Letters, Vol.. 67, Issue 20, pp. 2907-2909, 1995.

[34] Q. Wu and X.-C. Zhang, "Ultrafast electro-optic field sensors", Applied Physics Letters, Vol. 68, Isssue 12, pp. 1604-1606,1996.

[35] Q. Wu and X.-C. Zhang, "7 terahertz broadband GaP electrooptic sensor", Applied Physics Letters, Vol. 70, Issue14, , pp. 1784-1786, 1997

[36] R. Huber, A. Brodschelm, F. Tauser, and A. Leitenstorfer, "Generation and field-resolved detection of femtosecond electromagnetic pulses tunable up to 41 THz", Applied Physics Letters, Vol. 76, Issue 22 , pp. 3191-3193, 2000.

[37] C. Kübler, R. Hubera, S. Tübel and A. Leitenstorfer, "Ultrabroadband detection of multi-terahertz field transients with GaSe electro-optic sensors: Approaching the near infrared", Applied Physics Letters Vol. 85, Issue 16, , pp. 3360-3362, 2004

[38] J. Ahn, A. V. Efimov, R. D. Averitt, and A. J. Taylor, "Terahertz waveform synthesis via optical rectification of shaped ultrafast laser pulses", Optics Express, Vol. 11,Issue 20, pp. 2486-2496, 2003.

[39] M. Kreb, T. Loffler, M. D. Thomson, R. Dorner, H. Gimpel, K. Zrost, T. Ergler, R. Moshammer, U. Morgner, J. Ullrich and H. G. Roskos, "Determination of the carrier-envelope phase of fewcycle laser pulses with terahertz-emission spectroscopy", Nature Physics, Vol. 2, Issue 5, pp. 327-331, 2006.

[40] Nick C. J. van der Valk and Paul C. M. Planken, Anton N. Buijserd and Huib J. Bakker, "Influence of pump wavelength and crystal length on the phase matching of optical rectification", Journal of Optical Society of America B, Vol. 22, Issue 8, pp. 1714-1718, 2005

[41] T.Kampfrath,J. Nötzold and M. Wolf, "Sampling of broadband terahertz pulses with thick electro-optic crystals", Applied Physics Letters, Vol. 90, Issue 23, pp. 231113 (1-3), 2007

[42] G. Imeshev, M. E. Fermann, K. L. Vodopyanov, M. M. Fejer, X. $\mathrm{Yu}$ and J. S. Harris, D. Bliss and C. Lynch, "High-power source of $\mathrm{THz}$ radiation based on orientation-patterned GaAs pumped by a fiber", Optics Express. Vol. 14, Issue 10, pp. 4439-4444, 2006.

[43] Y. S. H. Leea, W. C. Hurlbut, K. L. Vodopyanov, M. M. Fejer, V. G. Kozlov, "Generation of multicycle terahertz pulses via optical rectification in periodically inverted GaAs structures", Applied Physics Letters, Vol. 89, Issue 18, pp. 181104 (1-3) ,2006.

[44] F. Blanchard, L. Razzari, H.-C. Bandulet, G. Sharma, R. Morandotti1, J.C. Kieffer, T. Ozaki, M. Reid, H. F. Tiedje, H. K. Haugen and F. A. Hegmann, "Generation of $1.5 \mu \mathrm{J}$ single-cycle terahertz pulses by optical rectification from a large aperture ZnTe crystal", Opticss Express Vol. 15, Issue 20, pp. 1321213220, 2007.

[45] J. A. Fülöp, L. Pálfalvi, S. Klingebiel, G. Almási, F. Krausz, S. Karsch, and J. Hebling, "Generation of sub- $m J$ terahertz pulses by optical rectification", Optics Letters, Vol. 37, Issue 4, pp. 557559,2012

[46] C. P. Hauri, C. Ruchert, C. Vicario and Fernando Ardana, "Strongfield single-cycle $\mathrm{THz}$ pulses generated in an organic crystal", Applied Physics Letters, Vol. 99, Issue 16, pp. 161116 (1-3), 2011.

[47] C. Ruchert, C. Vicario, C. P. Hauri, "Scaling submillimeter singlecycle transients toward megavolts per centimeter field strength via optical rectification in the organic crystal OH1", Optics Letters, Vol. 37, Issue 5, pp. 899-901, 2012. 
[48] C. Ruchert, C. Vicario and C. P. Hauri, "Spatiotemporal Focusing Dynamics of Intense Supercontinuum THz Pulses", Physical Review Letters, Vol. 110, Issue 12, pp. 123902 (1-5), 2013.

[49] C. Vicario, M. Jazbinsek, A. V. Ovchinnikov, O. V. Chefonov, S. I. Ashitkov, M. B. Agranat and C. P. Hauri, "High efficiency $\mathrm{THz}$ generation in DSTMS, DAST and OH1 pumped by Cr:forsterite laser", Optics Express, Vol. 23, Isue 4, pp. 4573-4580, 2015.

[50] J. Heblingr, A.G. Stepanov, G. Almási, B. Barta, J. Kuhl, "Tunable $\mathrm{THz}$ pulse generation by optical rectification of ultrashort laser pulses with tilted pulse fronts", Applied Physics B, . Phys. B, Vol. 78, Issue 5, pp. 593-599, 2004.

[51] J. Hebling, G. Almási and I. Z. Kozma, "Velocity matching by pulse front tilting for largearea THz-pulse generation", Optics Express, Vol. 10, Issue 21, pp. 1161-1166, 2002.

[52] A. G. Stepanov, J. Hebling and J. Kuhl, "Efficient generation of subpicosecond terahertz radiation by phase-matched optical rectification using ultrashort laser pulses with tilted pulse fronts", Applied Physics Letters, Vol. 83, Issue 15, pp. 3000-3002, 2003.

[53] K. L. Yeh, M. C. Hoffmann, J. Hebling and K. A. Nelson, "Generation of $10 \mu \mathrm{J}$ ultrashort terahertz pulses by optical rectification", Applied Physics Letters, Vol. 90, Issue 17, pp. $171121(1-3), 2007$.

[54] A. G. Stepanov, L. Bonacina, S. V. Chekalin, and J. P. Wolf, "Generation of $30 \mu \mathrm{J}$ single-cycle terahertz pulses at $100 \mathrm{~Hz}$ repetition rate by optical rectification", Optics Letters, Vol. 33, Issue 21, pp. 2497-2499, 2008.

[55] J. Hebling, K.L. Yeh, M. C. Hoffmann, B. Bartal and K. A. Nelson, "Generation of high-power terahertz pulses by tiltedpulse-front excitation and their application possibilities", Journal of Optical Society of America B, Vol. 25, Issue 7, pp. B6-B19, 2008

[56] J. A. Fulp, L. Palfalvi, G. Almasi and J. Hebling, "Design of highenergy terahertz sources based on optical rectification", Optics Express, Vol. 18, Issue 12, pp. 12311-12327, 2010.

[57] H. Hirori, A. Doi, F. Blanchard and K. Tanaka, "Single-cycle terahertz pulses with amplitudes exceeding $1 \mathrm{MV} / \mathrm{cm}$ generated by optical rectification in $\mathrm{LiNbO}_{3}$ ", Applied Physics Letters, Vol. 98, Issue 9, pp. 091106 (1-3), 2011.

[58] K. Ravi, W. R. Huang, S. Carbajo, X. Wu and F. Kärtner, "Limitations to THz generation by optical rectification using tilted pulse fronts", Optics Express", Vol. 22, No. 17 , pp. 20239-2251, 2014.

[59] K. Ravi, W. R. Huang, S. Carbajo, E. A. Nanni, D. N. Schimpf, E. P. Ippen, and F. X. Kärtner, "Theory of terahertz generation by optical rectification using tilted-pulse-fronts", Optics Express, Vol. 23, No. 4, pp. 5253-5276, 2015.

[60] S. W. Huang, E. Granados, W. R. Huang, K. H. Hong, L. E. Zapata and F. X. Kärtner, "High conversion efficiency, high energy terahertz pulses by optical rectification in cryogenically cooled lithium niobate", Optics Letters, Vol. 38, Issue 5, pp.796798, 2013

[61] W. R. Huang, S. W. Huang, E. Granados,, K. Ravi, K. H. Hong, L. E. Zapata and F. X. Kärtner, "Highly efficient terahertz pulse generation by optical rectification in stoichiometric and cryocooled congruent lithium niobate", Journal of Modern Optics, , Vol. 62 , Issue 18, pp. 1486-1493, 2015
[62] K. Ravi, M. Hemmer, G. Cirmi, F. Reichert, D. N. Schimpf, O. D. Mücke, and F. X. Kärtner, "Cascaded parametric amplification for highly efficient terahertz generation", Optics Letters, Vol. 41, Issue 16, pp. 3806-3809, 2016.

[63] K. Ravi, D. N. Schimpf, and F. X. Kärtner, "Pulse sequences for efficient multi-cycle terahertz generation in periodically poled lithium niobate", Vol. 24, Issue 22, pp. 25582-25607, 2016.

\section{AUTHORS PROFILE}

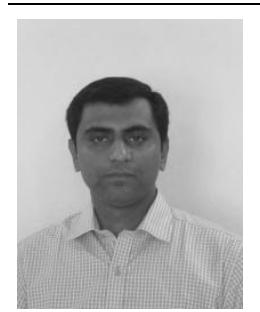

Dr. Sourabh Mukhopadhyay did his B. Sc. $(\mathrm{H})$ and M.Sc. in Physics from University of Calcutta and subsequently obtained Ph. D. in Physics from IIT Kharagpur in 2006. He was post doctoral fellow at University of Oxford during 2007-2008. Dr. Mukhopadhyay is working in West Bengal Education Service since 2006 and presently posted as assistant Professor at Jhargram Raj College. He has published more than 15 publications in reputed international journals and numerous international and national conference proceedings including CLEO USA. Present research interest of Dr. Mukhopadhyay includes ultrafast optics, $\mathrm{THz}$ sources and devices. 